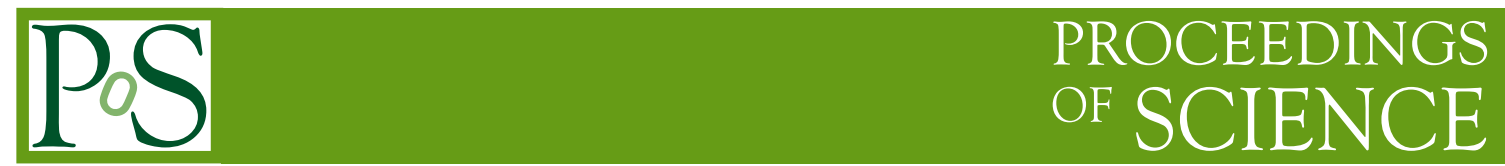

\title{
Low energy properties of color-flavor locked superconductors
}

\author{
Cristina Manuel* \\ Instituto de Fisica Corpuscluar (CSIC-U. de València) \\ E-mail: cristina.manueleific.uv.es
}

\begin{abstract}
We discuss some low energy properties of color-flavor locked (CFL) superconductors. First, we study how an external magnetic field affects their Goldstone physics in the chiral limit, stressing that there is a long-range component of the field that penetrates the superconductor. We note that the most remarkable effect of the applied field is giving a mass to the charged pions and kaons. By estimating this effect, we see that for values $\widetilde{e} \widetilde{B} \sim 2 f_{\pi} \Delta$, where $\Delta$ is the quark gap, and $f_{\pi}$ the pion decay constant, the charged Goldstone bosons become so heavy, that they turn out to be unstable. The symmetry breaking pattern is then changed, agreeing with that of the magnetic color-flavor locked (MCFL) phase, recently proposed in hep-ph/0503162. Finally, we discuss the physics of the superfluid phonon of the CFL phase, compare it with that of the phonon of a Bose-Einstein condensate, and discuss transport phenomena at low temperature. Astrophysical implications of all the above low energy properties are also commented.
\end{abstract}

29th Johns Hopkins Workshop on current problems in particle theory: strong matter in the heavens 1-3 August

Budapest

\footnotetext{
${ }^{*}$ Speaker.
} 


\section{Introduction}

In this talk I will touch two different aspects of the low energy physics of the color-flavor locked phase [1]. The first part is a continuation of Vivian de la Incera's seminar, on the subject of how a strong magnetic field influences color superconductivity. It is based on the joint collaboration with Efrain Ferrer and Vivian de la Incera [2]. In the second part, I will discuss transport phenomena at low temperature of the CFL and BEC superfluids, stressing similarities and differences. This is work done in collaboration with Antonio Dobado and Felipe Llanes-Estrada [3], and an on-going collaboration with Arnau Rios. Before entering into detailed discussions, let me explain you why those are interesting physical problems.

Studying the influence of a magnetic field on quark matter is not only an academic question. We believe that quark matter may occur in compact stars, either in the core of neutron stars, or in the form of quark stars. The real fact is that the highest magnetic fields in the Universe occur precisely in compact stars. Pulsars are believed to be neutron stars, and they stand magnetic fields in their surfaces in the range $B \sim 10^{12}-10^{14} \mathrm{G}$. Magnetars are a different kind of compact stars that stand higher magnetic fields, $B \sim 10^{14}-10^{15} \mathrm{G}$, while the value $B \sim 10^{16} \mathrm{G}$ is yet not discarded. There is however un upper theoretical limit to the magnetic field that a compact star may stand, arising after comparing the magnetic and gravitational energy, given as [4]

$$
B_{\max } \sim 1.4 \times 10^{18}\left(\frac{M}{M_{\odot}}\right)\left(\frac{10 \mathrm{~km}}{R}\right)^{2} G
$$

where $M$ and $R$ refer to the mass and radius of the star, respectively, and $M_{\odot}$ is the solar mass. If self-bound quark stars exist, this upper limit may go higher, though.

As discovered in [2], not only an applied magnetic field to the superconductor affects the quark gaps, thus affecting the equation of state of CFL quark matter. It also affects its low energy properties, as we will further discuss.

On the other hand, let me also insist on the relevance of transport phenomena in astrophysics. While with the equation of state one can determine the mass and radius of a star, with the transport coefficients one can study its cooling, and its vibrational and rotational properties. Thus, they are essential for detecting signatures of quark matter in compact stars in any of its possible phases.

It has been established that the viscosities put stringent tests to astrophysical models for very rapidly rotating stars, such as for millisecond pulsars. This is based on the existence of r(otational)mode instabilities in all relativistic rotating stars [5], which are only suppressed by sufficiently large viscosities. So the viscosities will allow one to discard unrealistic models for millisecond pulsars. There has not been many efforts in the literature to study transport phenomena in color superconducting quark matter, with very few exceptions $[6,7,8,3]$.

\section{Effects of a magnetic field in the low energy physics of the CFL phase}

For all the considerations to follow, we will neglect the effect of quark masses. In the absence of a magnetic field, three-flavor massless quark matter at high baryonic density is in the CFL phase [1]. Then the diquark condensates lock the color and flavor transformations, breaking both. The 
symmetry breaking pattern in the CFL phase is

$$
S U(3)_{C} \times S U(3)_{L} \times S U(3)_{R} \times U(1)_{B} \rightarrow S U(3)_{C+L+R} .
$$

There are only nine Goldstone bosons that survive to the Anderson-Higgs mechanism. One is a singlet, scalar mode, associated to the breaking of the baryonic symmetry, and the remaining octet is associated to the axial $S U(3)_{A}$ group, just like the octet of mesons in vacuum. At sufficiently high density, the anomaly is suppressed, and then one can as well consider the spontaneous breaking of an approximated $U(1)_{A}$ symmetry, and an additional pseudo Goldstone boson. We will ignore this effect, though.

Once electromagnetic effects are considered, the flavor symmetries of QCD are reduced, as only the $d$ and $s$ quarks have equal electromagnetic charges, $q=-e / 3$, while the $u$ quark has electromagnetic charge, $q=2 e / 3$. However, because the electromagnetic structure constant $\alpha_{\text {e.m. }}$. is so small, this effect is considered to be really tiny, a small perturbation, and one talks about approximated good flavor symmetries.

In the CFL phase there is a linear combination of the photon and a gluon that remains massless. The CFL diquarks are invariant under a $\widetilde{U}(1)_{\text {e.m. }}$ group, generated in flavor-color space by $\widetilde{Q}=$ $Q \times 1-1 \times Q$, where $Q$ is the electromagnetic charge generator. Then quarks of different flavors and colors all get integral value charges, given in terms of the charge of the electron $\tilde{e}=e \cos \theta$, where $\theta$ is the mixing angle.

The existence of this "rotated" electromagnetism implies, among other things, that an external magnetic field to the color superconductor will be able to penetrate it in the form of a "rotated" magnetic field, and this affects the pairing phenomena [2]. Furthermore, in the presence of a strong magnetic field one cannot consider the effects of electromagnetism as a small perturbation. Flavors symmetries are reduced, as both $U(1)_{\text {e.m. }}$ and $\widetilde{U}(1)_{\text {e.m. }}$ distinguish quark flavors. For sufficiently strong magnetic fields, quark matter is in the so-called magnetic CFL (MCFL) phase [2]. In the MCFL phase the symmetry breaking pattern is

$$
S U(3)_{C} \times S U(2)_{L} \times S U(2)_{R} \times U(1)_{A}^{(1)} \times U(1)_{B} \times U(1)_{\text {e.m. }} \rightarrow S U(2)_{C+L+R} \times \widetilde{U}(1)_{\text {e.m. }} .
$$

Here the symmetry group $U(1)_{A}^{(1)}$ is related to a current which is an anomaly free linear combination of $u, d$ and $s$ axial currents, and such that $U(1)_{A}^{(1)} \subset S U(3)_{A}$. The locked $S U(2)$ group corresponds to the maximal unbroken symmetry, such that it maximizes the condensation energy. The counting of broken generators, after taking into account the Anderson-Higgs mechanism, tells us that there are only five Goldstone bosons. As in the CFL case, one is associated to the breaking of the baryon symmetry; three Goldstone bosons are associated to the breaking of $S U(2)_{A}$, and another one associated to the breaking of $U(1)_{A}^{(1)}$. As before, if the effects of the anomaly could be neglected, there would be another pseudo Goldstone boson associated to the $U(1)_{A}$ symmetry.

An applied strong magnetic field, apart from modifying the value of some fermionic gaps, also affects the low energy properties of the color superconductor. There is a reduction in the number of Goldstone bosons, from nine to five. What happened to these four light particles of the CFL phase?

There is another question one may like to address. For which values of the magnetic field it is more appropriate to say that quark matter is in the CFL or in the MCFL phase? To answer properly this question, one should solve the whole set of gap equations for all values of the magnetic field, a rather difficult task that requires a numerical treatment. 
There is a fast way to answer to the above questions, which may allows us to give estimates for the transition values of magnetic field. We will study how an external magnetic field affects the effective field theory of the low energy degrees of freedom of the CFL phase.

\subsection{Effective field theory for the Goldstone bosons of the CFL phase in a magnetic field}

We review here how to construct the effective field theory for the light degrees of freedom of the CFL phase [9]. First one has to single out the phases of the diquark condensates

$$
X^{i a} \sim \varepsilon^{i j k} \varepsilon^{a b c}\left\langle\psi_{L}^{b j} \psi_{L}^{c k}\right\rangle^{*}, \quad Y^{i a} \sim \varepsilon^{i j k} \varepsilon^{a b c}\left\langle\psi_{R}^{b j} \psi_{R}^{c k}\right\rangle^{*}
$$

where $a, b, c$ denote flavor indices, $i, j, k$ denote color indices, and $L / R$ denote left/right chirality, respectively. Under an $S U(3)_{C} \times S U(3)_{L} \times S U(3)_{R}$ rotation, the above fields transform as

$$
X \rightarrow U_{L} X U_{C}^{\dagger}, \quad Y \rightarrow U_{R} Y U_{C}^{\dagger} .
$$

The combination defined by

$$
\Sigma=X Y^{\dagger}
$$

is a color singlet, which under $S U(3)_{C} \times S U(3)_{L} \times S U(3)_{R}$ transforms as

$$
\Sigma \rightarrow U_{L} \Sigma U_{R}^{\dagger}
$$

One parametrizes the unitary matrix $\Sigma$ as

$$
\Sigma=\exp \left(i \frac{\Phi}{f_{\pi}}\right), \quad \Phi=\phi^{A} T^{A}
$$

where $T^{A}$ are the $S U(3)$ generators, defining the Goldstone fields as in vacuum

$$
\Phi=\left(\begin{array}{ccc}
\pi_{0}+\frac{1}{\sqrt{3}} \eta_{8} & \sqrt{2} \pi^{+} & \sqrt{2} K^{+} \\
\sqrt{2} \pi^{-} & -\pi_{0}+\frac{1}{\sqrt{3}} \eta_{8} & \sqrt{2} K^{0} \\
\sqrt{2} K^{-} & \sqrt{2} \bar{K}^{0} & \frac{-2}{\sqrt{3}} \eta_{8}
\end{array}\right) .
$$

For energy scales $E \ll 2 \Delta$, where $\Delta$ is the quark gap, the Lagrangian in the chiral limit for the Goldstone fields is pretty much the same as in vacuum, with the only exception that temporal and spatial derivatives are not related, due to the lack of Lorentz symmetry at finite density

$$
\mathscr{L}=\frac{f_{\pi}^{2}}{4}\left(\operatorname{Tr}\left(\partial_{0} \Sigma \partial_{0} \Sigma^{\dagger}\right)-v_{\pi}^{2} \operatorname{Tr}\left(\partial_{i} \Sigma \partial_{i} \Sigma^{\dagger}\right)\right) .
$$

For asymptotic large values of the chemical potential $\mu$, the parameters appearing in (2.9) can be computed, and read [10]

$$
f_{\pi}^{2}=\frac{21-8 \ln 2}{18} \frac{\mu^{2}}{2 \pi^{2}}, \quad v_{\pi}=\frac{1}{\sqrt{3}} .
$$

We have ignored in all the above considerations the Goldstone boson of baryon symmetry breaking, which will deserve a separated treatment in Section 3. 
Electromagnetic interactions can be introduced taking into account, as commented in the previous section, that they do not respect the whole $S U(3)_{L} \times S U(3)_{R}$ symmetry. To see which are the new allowed terms in the low energy effective field theory one proceeds as follows. The quark charge matrix $Q$ represents an explicit breaking term of the $S U(3)_{L} \times S U(3)_{R}$ symmetry in the QCD Lagrangian. In order to see its effects in the low energy physics, one treats $Q$ as a spurion field, whose vacuum expectation value is the responsible of the symmetry breaking. To restore the symmetry one has to introduce left and right charge matrices, which transform as

$$
Q_{L} \rightarrow U_{L} Q_{L} U_{L}^{\dagger}, \quad Q_{R} \rightarrow U_{R} Q_{R} U_{R}^{\dagger},
$$

under a $S U(3)_{L} \times S U(3)_{R}$ transformation.

Then, the derivatives in Eq. (2.9) should become covariant derivatives

$$
\partial_{\mu} \Sigma \rightarrow \widetilde{D}_{\mu} \Sigma=\partial_{\mu} \Sigma-i \tilde{e} Q_{L} \tilde{A}_{\mu} \Sigma+i \tilde{e} \Sigma Q_{R} \tilde{A}_{\mu}
$$

Using simple considerations one can see that also a term of the sort $\operatorname{Tr}\left(Q_{L} \Sigma Q_{R} \Sigma^{\dagger}\right)$ is allowed by the symmetries in the Lagrangian. Thus, it reads [12]

$$
\begin{aligned}
\mathscr{L}= & \frac{\widetilde{\varepsilon}}{2} \widetilde{\mathbf{E}}^{2}-\frac{1}{2 \widetilde{\lambda}} \widetilde{\mathbf{B}}^{2} \\
& +\frac{f_{\pi}^{2}}{4}\left[\operatorname{Tr}\left(\widetilde{D}_{0} \Sigma \widetilde{D}_{0} \Sigma^{\dagger}\right)-v_{\pi}^{2} \operatorname{Tr}\left(\widetilde{D}_{i} \Sigma \widetilde{D}_{i} \Sigma^{\dagger}\right)\right]+C \operatorname{Tr}\left(Q \Sigma Q \Sigma^{\dagger}\right),
\end{aligned}
$$

Here, again, we have taken into account that spatial and temporal components of $\widetilde{F}_{\mu v}$ go separately. The value of $\widetilde{\varepsilon} \approx 1+\frac{2}{9 \pi^{2}} \frac{\tilde{e}^{2} \mu^{2}}{\Delta^{2}}$, and $\widetilde{\lambda} \approx 1$ are obtained after integrating out the high energy fermionic modes [12], and represent an effective dielectric constant and magnetic susceptibility, respectively.

If one uses the value of $Q=e(2 / 3,-1 / 3,-1 / 3)$, one easily recognizes that the last term of (2.13) is a mass term for the charged Goldstone bosons, plus additional meson contact interactions. More particularly, one finds

$$
M_{\pi^{ \pm}}^{2}=M_{K^{ \pm}}^{2}=\frac{2 \tilde{e}^{2} C}{f_{\pi}^{2}} .
$$

The value of $C$ could be in principle computed at high baryonic density [11]. However, it requires to evaluate a complicated three-loop diagram. An estimate of the diagram [11], together with dimensional analysis, suggests that $M_{\pi^{ \pm}}^{2}=M_{K^{ \pm}}^{2} \sim \tilde{e}^{2} \Delta^{2}$.

In writing Eq.(2.13) one assumes a particular power counting. The chiral Lagrangian is written as an expansion in momenta, and here only the lowest order terms have been kept. With this counting, one assumes $p^{2} / f_{\pi}^{2} \sim \tilde{e}^{2}$.

We will now assume the existence of an external weak magnetic field $\widetilde{\mathbf{B}}^{\text {ext }}$, and see the modifications in the chiral Lagrangian (2.13). The presence of an external field brings a new dimensional scale in the problem. For the low energy theory to make sense, one then has to assume that the field is such that $p^{2} \sim \tilde{e} \widetilde{B}^{\text {ext }}$.

One can get the effective field theory for the Goldstone bosons in the presence of the magnetic field from (2.13), simply by splitting all the vector gauge fields into a background part and a fluctuating part

$$
\tilde{A}_{\mu} \rightarrow \tilde{A}_{\mu}^{\mathrm{ext}}+\tilde{A}_{\mu}
$$


where, for example, one can take $\tilde{A}_{\mu}^{\text {ext }}=\left(0,0, x \tilde{B}^{\text {ext }}, 0\right)$ to reproduce a magnetic field pointing in the $z$ direction.

With the established power counting rules and symmetry transformation of $Q_{L / R}$, it is not difficult to see new terms entering into the low energy Lagrangian. We will however now neglect the effect of electromagnetic fluctuations, and consider the direct effects of the external magnetic field, assuming $\tilde{e}^{2} \ll p^{2} / f_{\pi}^{2} \sim \tilde{e} \widetilde{B}^{\text {ext }} / f_{\pi}^{2}$. It is not difficult to see that a new term in the chiral Lagrangian is allowed, namely

$$
D\left(\widetilde{\mathbf{B}}^{\mathrm{ext}} \cdot \widetilde{\mathbf{B}}^{\mathrm{ext}}\right) \operatorname{Tr}\left(Q \Sigma Q \Sigma^{\dagger}\right)
$$

As before, this essentially represents a mass term for the charged Goldstone bosons

$$
M_{\pi^{ \pm}}^{2}=M_{K^{ \pm}}^{2}=\frac{\left(\tilde{e} \widetilde{B}^{\mathrm{ext}}\right)^{2}}{f_{\pi}^{2}} D
$$

plus additional contact interactions. Here, $D$ should be computed from the microscopic theory. It is an adimensional number, and for the power counting to make sense, it should be of order $O(1)$.

All these considerations assume a weak magnetic field, so that the power counting rules are valid. But one can imagine increasing the value of the external field. As suggested by Eq. (2.17), this would imply an increase of the charged Goldstone boson masses. By strengthening the field more and more, the charged pions and kaons become heavier and heavier. It will reach one point when they will become unstable. This will happen when they have a mass of order $2 \Delta$, the energy necessary to break a Cooper pair of quarks. If we extrapolate the value of the mass of the charged Goldstone bosons from weak to strong fields, this happens when

$$
\tilde{e} \tilde{B}^{\mathrm{ext}} \sim 2 f_{\pi} \Delta
$$

assuming that $D$ is of order $O(1)$. To get an idea of the values of the magnetic field we are getting, we will use the value of $f_{\pi}$ of Eq. (2.10), for $\mu \sim 400 \mathrm{MeV}$, and $\Delta \sim 25 \mathrm{MeV}$. One gets $\tilde{e} \tilde{B}^{\mathrm{ext}} \sim 5 \cdot 10^{16} \mathrm{G}$. For those values of the magnetic field, the system will be formed by only five neutral Goldstone bosons. This is precisely the number predicted from the symmetry considerations explained in the previous subsection. We can deduce that for the above values of the magnetic field, it will more appropriate to say that quark matter is in the MCFL phase.

\section{Transport in the CFL phase at low temperature}

The BCS theory explains the main phenomenological properties of electromagnetic superconductors, such as the Meissner effect, and the appearance of an energy gap $\Delta$ in the quasiparticle spectrum. It also explains the reason why superconductors are both good heat conductors and superfluids, with exponentially suppressed transport coefficients $\sim \exp [-\Delta / T]$ for low temperatures $T \ll \Delta$.

Should we expect the transport coefficients in a color superconductor to behave as in an electromagnetic superconductor? Naively, one would say so, but the answer is slightly more elaborated. Because the hydrodynamic regime of a system depends on the low energy spectrum of the theory, a careful study of the low energy properties of every color superconducting phase is needed before evaluating transport coefficients. 
Transport properties in the CFL phase of QCD at low $T$ are not dominated by the quarks, which certainly give a contribution of the sort $\sim \exp [-\Delta / T]$, as the electrons in an electromagnetic superconductor. There are many light degrees of freedom in the CFL phase whose contribution is bigger: nine Goldstone bosons (or five in the presence of a strong magnetic field!), and the "rotated" photon. In a possible CFL quark star at finite temperature leptons are thermally excited, so one may also expect to find electrons. In principle, neutrinos should as well be considered for transport. However, for the astrophysical applications we have in mind, in the temperature regime we are going to consider all the neutrinos have already escaped from the star, as it is deduced from their mean free path $[13,14]$.

Chiral symmetry is not an exact symmetry of QCD. Therefore, the associated (pseudo) Goldstone bosons are massive. Their masses are estimated to be in the range of the tens of $\mathrm{MeV}$ [10]. At sufficiently low temperatures $\left(T \ll \Delta, m_{\pi}\right.$ ) their contribution to the viscosities is Boltzmann suppressed, and transport is dominated by the lightest particles. In particular, the highest contribution [8] is given by the Goldstone boson associated to baryon symmetry breaking, the superfluid phonon, which remains massless. There is also a contribution of the in-medium electromagnetism, but this turns out to be negligible. In Ref. [3] the mean free path and shear viscosity for the superfluid phonon were computed, finding many similarities with those of the superfluid phonon in $\mathrm{He}^{4}$. Here we will try to explain why this is so. For all the considerations in this section, we will neglect the effects of an external magnetic field.

\subsection{Effective field theory for the superfluid phonon}

Superfluidity in $\mathrm{He}^{4}$ is understood as a consequence of Bose-Einstein condensation (BEC). The BEC and CFL superfluids have in principle very little in common - the first system is bosonic and non-relativistic, while the second one is fermionic and relativistic. However, in the two systems there is a spontaneous breaking of a global $U(1)$ symmetry, associated to particle density in one case, to baryon charge in the other. Hence, there is a massless collective mode or Goldstone boson in the two cases. We will call it in the two cases superfuid phonon. ${ }^{1}$

For the two systems one can use the techniques of effective field theory to learn about the selfinteractions of the superfluid phonons. The low energy Lagrangian is constructed using a power expansion in momenta, taking into account properly the underlying unbroken symmetries of the system. In a BEC the phonon $\varphi$ appears as the phase of the boson condensate. Galilean invariance implies that the Lagrangian is expressed in terms of powers of $\left(\partial_{0} \varphi-\frac{(\nabla \varphi)^{2}}{2 m}\right)$ [15]. In the CFL system the phonon appears as a phase of the diquark condensate. Lorentz invariance would be a symmetry of the system if it were not for the presence of the chemical potential $\mu$. One can treat $\mu$ as a spurion field, and then one sees that effective Lagrangian for the phonon in this case can be organized as a power expansion in $D_{\mu} \varphi=\partial_{\mu} \varphi-A_{\mu}$, where $A_{\mu}=(\mu, 0)$ [18].

The specific coefficients of the effective Lagrangian in each case should be determined from the microscopic theory by a matching procedure. However, this matching actually can only be performed in the weak coupling regime of the theory. When the system is not weakly coupled, one

\footnotetext{
${ }^{1}$ The name of phonon was chosen by Landau, when he "guessed" that a collective mode with a linear dispersion relation was needed to explain the superfluid behavior of $\mathrm{He}^{4}$. More frequently now "phonon" is used to denote the Goldstone boson associated to the spontaneous breaking of a translational symmetry, as it occurs in crystals.
} 
can still write down an effective field theory for the low energy degrees of freedom, and expect to have an experimental result to determine the values of those coefficients. This is the situation that occurs for the mesons of QCD at vacuum, for example, where the value $f_{\pi}$ is only determined experimentally.

In the CFL case the matching procedure can be performed for very large densities, the result is [18]

$$
\mathscr{L}_{\text {eff }}^{\mathrm{CFL}}=\frac{3}{4 \pi^{2}}\left[\left(\partial_{0} \varphi-\mu\right)^{2}-(\nabla \varphi)^{2}\right]^{2}+\cdots
$$

For a BEC superfluid the effective phonon Lagrangian reads [17]

$$
\mathscr{L}_{\text {eff }}^{\mathrm{BEC}}=c_{1}\left(\partial_{0} \varphi-\frac{(\nabla \varphi)^{2}}{2 m}\right)+\frac{c_{2}}{2}\left(\partial_{0} \varphi-\frac{(\nabla \varphi)^{2}}{2 m}\right)^{2}+\cdots
$$

For a weakly interacting bosonic system the parameters $c_{1}$ and $c_{2}$ can be computed from the microscopic theory [17], and are given in terms of the bosonic particle density $c_{1}=n_{0}$, and the $s$-wave scattering length $a, c_{2}=1 / 4 \pi a$. Let us mention that a similar effective field theory, keeping not only the Goldstone boson, but also the quantum fluctuations of the bosonic density has been derived in Refs. [15, 16]. Eq. (3.2) is obtained from those if one further integrates out the density fluctuations.

We re-scale $\varphi$ to get the standard kinetic term in both (3.1) and (3.2) to obtain

$$
\mathscr{L}_{\mathrm{eff}}^{\mathrm{CFL}}=\frac{1}{2}\left(\partial_{0} \phi\right)^{2}-\frac{v^{2}}{2}(\nabla \phi)^{2}-\frac{\pi}{9 \mu^{2}} \partial_{0} \phi\left(\partial_{\mu} \phi \partial^{\mu} \phi\right)+\frac{\pi^{2}}{108 \mu^{4}}\left(\partial_{\mu} \phi \partial^{\mu} \phi\right)^{2},
$$

and

$$
\mathscr{L}_{\mathrm{eff}}^{\mathrm{BEC}}=\frac{1}{2}\left(\partial_{0} \phi\right)^{2}-\frac{v^{2}}{2}(\nabla \phi)^{2}-\frac{1}{2 m \sqrt{c_{2}}} \partial_{0} \phi(\nabla \phi)^{2}+\frac{1}{8 m^{2} c_{2}}(\nabla \phi)^{4}
$$

respectively. In the two cases, we have omitted a total time derivative, which is only needed to study vortex configurations. To lowest order in momenta, the superfuid phonon dispersion relation reads

$$
E_{p}=v p
$$

where, in the two cases, $v$ is given by the speed of sound of the system. More particularly, in the CFL case $v^{2}=1 / 3$, while for the BEC $v^{2}=c_{1} / m c_{2}$. It is curious to note that even in a nonrelativistic $\mathrm{BEC}$ the physics of the phonon is relativistic.

At low temperature the dissipation in both the CFL and BEC superfluids will be dominated by the thermal properties of the superfluid phonons, such as damping, scattering rates, mean free paths. These can be computed from the effective field theories displayed above.

Let us first see how the one-loop thermal physics is obtained. We will use the imaginary time formalism (ITF) for the evaluation of the Feynman diagrams. There are two different diagrams that contribute to the one-loop self-energy, see Fig. 1. For external momentum $P=\left(p_{0}, \mathbf{p}\right)=(i \omega, \mathbf{p})$, these are

$$
\Pi^{(a)}(P)=\int \frac{d^{d} K}{(2 \pi)^{d}} H(P, K) S(K)
$$

and

$$
\Pi^{(b)}(P)=\int \frac{d^{d} K}{(2 \pi)^{d}}(F(P, K) S(K) S(P-K))
$$




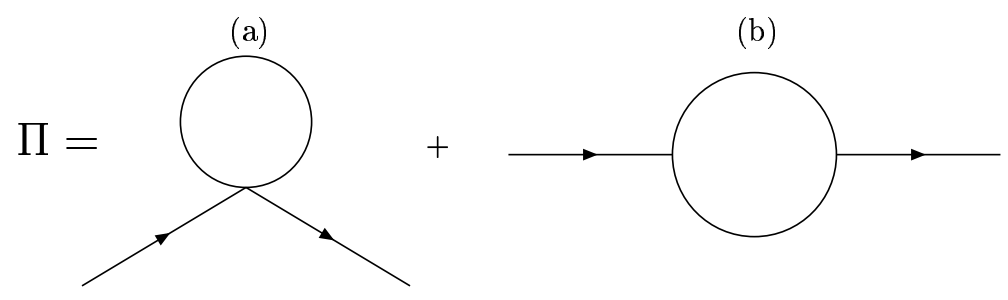

Figure 1: One-loop contributions to the phonon self-energy.

respectively, where $S$ is the phonon propagator. In ITF, with momentum $K=\left(k_{0}, \mathbf{k}\right)=\left(i \omega_{n}, \mathbf{k}\right)$, it reads

$$
S(K) \equiv \frac{1}{\omega_{n}^{2}+E_{k}^{2}}
$$

where $\omega_{n}=2 \pi n T$, with $n \in \mathscr{Z}$, is a bosonic Matsubara frequency. The functions $H$ and $F$ are slightly different for the CFL and BEC superfluids. More particularly, in the first case, one has

$$
\begin{gathered}
H^{\mathrm{CFL}}(P, K)=\frac{\pi^{2}}{27 \mu^{4}}\left(2(K \cdot P)^{2}+P^{2} K^{2}\right), \\
F^{\mathrm{CFL}}(P, K)=\frac{4 \pi^{2}}{81 \mu^{4}}\left(p_{0}\left(2 K \cdot P-K^{2}\right)+k_{0}\left(P^{2}-2 K \cdot P\right)\right)^{2},
\end{gathered}
$$

while for the BEC

$$
\begin{gathered}
H^{\mathrm{BEC}}(P, K)=\frac{1}{m^{2} c_{2}}\left(2(\mathbf{k} \cdot \mathbf{p})^{2}+p^{2} k^{2}\right) \\
F^{\mathrm{BEC}}(P, K)=\frac{1}{m^{2} c_{2}}\left(p_{0}\left(2 \mathbf{k} \cdot \mathbf{p}-k^{2}\right)+k_{0}\left(p^{2}-2 \mathbf{k} \cdot \mathbf{p}\right)\right)^{2} .
\end{gathered}
$$

The BEC functions are simply obtained by replacing the four momenta by three momenta of the CFL functions, apart from an overall factor.

Since the phonon is a Goldstone boson, and thermal effects do not represent an explicit breaking of the $U(1)$ symmetry, its self-energy should vanish at $P=0$. It is actually easy to check that this holds at one-loop level

$$
\Pi^{(a)}(P=0)=\Pi^{(b)}(P=0)=0,
$$

so no thermal mass is generated. This property of the self-energy should hold to all orders in perturbation theory.

The physics associated to the superfluid phonon is peculiar. Even if at finite $T$ almost all particles attain a thermal mass, this is not so for the phonon, as thermal effects do not represent a violation of the $U(1)$ symmetry. The superfluid phonon suffers Landau damping, which for $p_{0}, p \ll T$ reads

$$
\operatorname{Im} \Pi\left(p_{0}, \mathbf{p}\right) \approx \frac{8 \pi^{5}}{1215} \frac{T^{4}}{v^{8} \mu^{4}} \frac{p_{0}^{3}}{p} \Theta\left(v^{2} p^{2}-p_{0}^{2}\right) .
$$

for the CFL superfluid, while for the BEC superfluid one gets

$$
\operatorname{Im} \Pi\left(p_{0}, \mathbf{p}\right) \approx-\frac{\pi^{3}}{6 m^{2}} \frac{T^{4}}{v^{8} c_{2}} \frac{p_{0}^{3}}{p} \Theta\left(v^{2} p^{2}-p_{0}^{2}\right) .
$$


Evaluating this imaginary time on-shell, one finds the superfluid phonon lifetime in the thermal bath. In the last case, one matches old results obtained using different techniques [19].

In the same way, one can start computing the mean free path associated to different phononphonon collisions. Large-angle collisions in the two cases scale with the temperature as $\sim 1 / T^{9}$. Small-angle collisions also scale in the two cases as $\sim 1 / T^{5}$. With the explanations in this Section, now one may understand the claims of Ref. [3]. There it was mentioned that the superfluid phonon in the CFL system had the same temperature dependence in the damping, and also in the mean free path for small and large angle collisions that in superfluid $\mathrm{He}^{4}$, in its low temperature regime [20].

Still, there is a difference in the shear viscosity of the two systems, as we explain in the following subsection.

\subsection{Shear viscosity in the phonon fluid}

Landau proposed the two fluid model of superfluidity [21], deriving the corresponding nonrelativistic hydrodynamic equations. He incorporated the effects of dissipation, taking into account viscosity. It is noteworthy that in the superfluid there is one shear viscosity coefficient, but two bulk viscosities, associated to the fact that there are two fluid velocities. The relativistic version of these hydrodynamic equations are slightly more involved. They have been worked out in Ref. [22].

There are several subtle points in the computation of transport coefficient in the low temperature regime of these superfluids. Here we will only discuss the shear viscosity computation.

Prior to the computation of a transport coefficient, one has to evaluate the mean free path of the collisions which are relevant for the corresponding transport phenomena. Shear viscosity describes the relaxation of the momentum components perpendicular to the direction of transport, and it usually requires large-angle collisions. However, the computation of this transport coefficient becomes complicated by the fact that the differential cross section of binary collisions mediated by phonon exchange is divergent for small-angle collisions. This is the typical Coulomb-Rutherford collinear divergence induced by massless exchange. In an ordinary scalar theory such a divergence does not appear, as a thermal mass is generated even if the boson is massless in vacuum. But the phonon remains massless at finite temperature. In Ref. [3] we have suggested that the divergence is regulated by the finite width of the phonon, or more precisely, by Landau damping, a process only occurring in a thermal bath. After regularization, we find that small-angle processes have a shorter mean free path than large-angle ones. This suggests that they might be more relevant for viscosity, as a large-angle collision can always be achieved by the addition of many small-angle ones.

To compute the shear viscosity one solves the Boltzmann equation, linearizing it for small departures from equilibrium. Then one finds that zero mode occurs in the collision operator for small-angle collisions. This happens both for the BEC superfluid [20], as for the CFL superfuid [3]. These zero modes act on the direction of suppressing the small-angle collision contribution to the shear viscosity.

In the CFL superfluid, this suppression is very severe. Could this be changed by considering higher order effects, not contained in the lowest low energy Lagrangian (3.3)? In Ref. [23] the superfluid phonon dispersion relation was computed to higher order, finding

$$
\omega(k)=v k\left[1-\frac{11}{540} \frac{k^{2}}{\Delta^{2}}+\mathscr{O}\left(\frac{k^{4}}{\Delta^{4}}\right)\right] .
$$


As $k$ is increased, the phonons move slower, with the tendency of suppressing collinear splitting (a phonon cannot decay into two phonons of larger joint energy), and then the small-angle processes are kinematically forbidden. We then found a shear viscosity coefficient [3]

$$
\eta=1.3 \cdot 10^{-4} \cdot \frac{\mu^{8}}{T^{5}} \mathrm{MeV}^{3}
$$

In superfluid $\mathrm{He}^{4}$, the value of mean free path for shear viscosity is however compatible with that of small-angle collisions [20]. This led to Maris to propose a different dispersion relation for the superfluid phonon [20]

$$
\omega(k)=v k[1+g(k)] .
$$

with $g(k)$ a positive function that tends to zero for $k \rightarrow 0$. In this way, phonons with high momenta move faster than those of low momenta, and can decay into two slower phonons. Maris proposed different functions $g(k)$ which more or less fit the experimental value of the shear viscosity, although the choices seem to be rather arbitrary, and not physically motivated.

Effective field theory techniques should be more appropriated to tackle the computation of transport coefficients in a BEC at low temperature. In a weakly coupled system, $g(k)$ could be computed from the microscopic theory to a high level of accuracy. In a strongly coupled system, probably $g(k)$ can be determined experimentally. We hope to report in the future about this approach to transport phenomena in a BEC.

\section{Conclusions}

Determination of the low energy properties are essential for any study of signatures of color superconductivity in astrophysical scenarios.

We have stressed that a strong magnetic field, such as those that might be found in the core of some highly magnetized compact stars, has dramatic consequences for the low energy properties of a color superconductor, through the disappearance of low energy modes from the spectrum. The influence of this fact on some macroscopic properties of the superconductor has still to be analyzed.

We have presented a computation of the shear viscosity in the CFL phase. For the analysis of r-mode instabilities bulk viscosity coefficients are also necessary, and have not yet been computed.

For astrophysical applications, we should emphasize the following. At sufficiently low $T$ the phonon mean free path would exceed the radius of a compact star. We can give a crude estimate of the temperature when this will occur, simply by considering the equation

$$
R<L \sim \mu^{4} / T^{5}
$$

If the quark chemical potential is of order $\mu \sim 500 \mathrm{MeV}$, and we consider $R \sim 10 \mathrm{~km}$, we find that for $T<0.06 \mathrm{MeV}$ superfluid phonons do not scatter within the star. Transport coefficients could then be dominated by the tiny contribution of the in-medium electromagnetism, but an evaluation of the photon mean free path also shows that for $T \sim 0.02 \mathrm{MeV}$ it also exceeds the radius of the star. Below that temperature, CFL quark matter in the star would behave as a perfect superfluid, showing then no dissipation, as a hydrodynamical description of the phonon and electron fluids would be meaningless. 
In a rotating superfluid there are vortices. To study the rotational properties of a hypothetical CFL quark star, one cannot obviate that fact. In view of our results, the analysis of r-mode instabilities of a CFL quark star should then be redone, taking into account both the temperature regime of the star, and the vortex dynamics of the CFL phase.

\section{Acknowledgments}

I would like to thank the organizers of this conference for giving me the opportunity to participate in this vivid meeting, as well as my collaborators E. Ferrer, V. de la Incera, A. Dobado, F. Llanes-Estrada and A. Rios. This work has been supported by MEC under grant No. FPA200400996.

\section{References}

[1] M. Alford, K. Rajagopal and F. Wilczek, "Color-flavor locking and chiral symmetry breaking in high density,” Nucl. Phys. B537, 443 (1999). [arXiv:hep-ph/9804403].

[2] E. J. Ferrer, V. de la Incera and C. Manuel, "Magnetic color flavor locking phase in high density QCD,” Phys. Rev. Lett. 95, 152002 (2005) [arXiv:hep-ph/0503162].

[3] C. Manuel, A. Dobado and F. J. Llanes-Estrada, "Shear viscosity in a CFL quark star,", JHEP09, 076 (2005) [arXiv:hep-ph/0406058].

[4] D. Lai, “Matter in Strong Magnetic Fields," Rev. Mod. Phys. 73, 629 (2001) [arXiv:astro-ph/0009333].

[5] N. Andersson, "A new class of unstable modes of rotating relativistic stars," Astrophys. J. 502, 708 (1998). [arXiv:gr-qc/9706075].

[6] J. Madsen, "Probing strange stars and color superconductivity by r-mode instabilities in millisecond pulsars,” Phys. Rev. Lett. 85, 10 (2000).[arXiv:astro-ph/9912418].

[7] D. F. Litim and C. Manuel, "Transport theory for a two-flavor color superconductor," Phys. Rev. Lett. 87, 052002 (2001). [arXiv:hep-ph/0103092].

[8] I. A. Shovkovy and P. J. Ellis, "Thermal conductivity of dense quark matter and cooling of stars," Phys. Rev. C66, 015802 (2002). [arXiv:hep-ph/0204132].

[9] R. Casalbuoni and R. Gatto, "Effective theory for color-flavor locking in high density QCD," Phys. Lett. B464 (1999) 111 [arXiv:hep-ph/9908227].

[10] D. T. Son and M. A. Stephanov, "Inverse meson mass ordering in color-flavor-locking phase of high density QCD,” Phys. Rev. D61, 074012 (2000) [arXiv:hep-ph/9910491]; Phys. Rev. D62, 059902 (2000) [hep-ph/0004095].

[11] C. Manuel and M. H. Tytgat, "Sum rules in the CFL phase of QCD at finite density," Phys. Lett. B 501, 200 (2001) [arXiv:hep-ph/0010274].

[12] D. F. Litim and C. Manuel, "Photon self-energy in a color superconductor," Phys. Rev. D 64, 094013 (2001) [arXiv:hep-ph/0105165].

[13] S. Reddy, M. Sadzikowski and M. Tachibana, "Neutrino rates in color flavor locked quark matter," Nucl. Phys. A 714, 337 (2003) [arXiv:nucl-th/0203011].

[14] P. Jaikumar, M. Prakash and T. Schafer, "Neutrino emission from Goldstone modes in dense quark matter," Phys. Rev. D 66, 063003 (2002) [arXiv:astro-ph/0203088]. 
[15] V. N. Popov, "Functional Integrals in Quantum Field Theory and Statistical Physics," (Reidel, Dordrecht, 1983).

[16] W. V. Liu, “Effective field theory approach to Bose-Einstein condensation,” Int. J. Mod. Phys. B 12 (1998) 2103.

[17] J. O. Andersen, "Effective Field Theory for Goldstone Bosons in Nonrelativistic Superfluids," [arXiv:cond-mat/0209243].

[18] D. T. Son, “Low-energy quantum effective action for relativistic superfluids,” [arXiv:hep-ph/0204199].

[19] P. C. Hohenberg and P. C. Martin, "Microscopic theory of superfluid helium, Ann. Phys. (N.Y.) 34, 291 (1965).

[20] H. J. Maris, "Hydrodynamics of Superfluid Helium below 0.6 K. Viscosity of the Normal Fluid", Phys. Rev. A 8, 1980 (1973).

[21] L. Landau and Lifschitz, "Statistical Physics" vol. 5;"Fluid Mechanics” vol. 6, Prentince Hall, New Jersey.

[22] V. V. Lebedev and I. M. Khalatnikov, Zh. Eksp. Teor. Fiz. 56, 1601 (1982); B. Carter and I. M. Khalatnikov, Rev. Math. Phys. 6, 277 (1994)

[23] K. Zarembo, “Dispersion laws for Goldstone bosons in a color superconductor,” Phys. Rev. D 62, 054003 (2000) [arXiv:hep-ph/0002123]. 